\title{
Polygon "Simeiz-Katsively" - fluctuations in the local deformations and insolation the Earth's surface
}

\author{
Larisa Volvach $^{1, *}$, Galina Kurbasova ${ }^{1}$, Andrej Dmytrotsa ${ }^{1}$, and Dmitry Neyachenko ${ }^{1}$ \\ ${ }^{1}$ Radio Astronomy and Geodynamics Department, Crimean Astrophysical Observatory RAS, Katsively
}

\begin{abstract}
Discovered the effects of regular solar and geodynamic fluctuations in satellite data on the insolation falling on the earth's surface at the point of the Kara-Dag for the period 1983.5 in 2005.5 years. The parameters of the seasonal component are determined. Possible reasons for the increase in the amplitude of the seasonal component are discussed.
\end{abstract}

\section{Introduction}

To explore emerging and developing deformation processes, movements of lithospheric plates are created geodynamic polygons, experimental equipment, which implements the comprehensive observational techniques of radio interferometry with very long base (VLBI), laser ranging to artificial satellites (LRS) satellite systems GPS and GLONASS. One of the main objectives of helio-geodynamic research based on geodetic and space-based measurements is to create and maintain catalogs and maps the rates of change of deformation of the earth's surface.

The Crimean Peninsula is located near the border of the Eurasian lithospheric plate. Deformation and movement of the plate over long periods of time and have had currently effect on improvement of processes inside the earth (earthquakes, volcanoes, mudslides). The activation inside the earth processes is indicated by anomalies in the displacement of the surface of observation points.

The influence of external and internal earth processes on the climate and ecology of Crimea manifests itself in different ways on different time scales. Therefore, the description of longterm changes and prediction of geophysical and climatic characteristics of the Peninsula are possible on continuous ground-based observations over many years.

\section{Monitoring of deformation processes}

Recently, the relevance of studying the geo-geodynamic processes occurring in near-surface geological structures in local territories, which are characteristic of cities and areas of large engineering structures, has sharply increased. At the same time, special attention in the preparation of long-term local forecasts is given to the use of satellite climatic parameters subject to heliogeodynamic influences $[1,2]$. Analysis of changes in the structure of long-term continuous series of observations establishes the presence of anomalies.

\footnotetext{
*Corresponding author: volvach@,bk.ru
} 
To explore emerging and developing deformation processes, movements of lithospheric plates are created geodynamic polygons, equipped with high-precision experimental equipment, which is implemented to conduct comprehensive observational methods radiointerferometry with very long base (VLBI), laser ranging to artificial satellites (LRS) satellite systems GPS and GLONASS.

Long-term observational program, involving dozens of countries and many international and national organizations, based on the achievements of the Global navigation satellite system GNSS: GPS (USA) and GLONASS (Russia).

On the geodynamic polygon "Simeiz-Katsively" observations have been carried out by several methods (VLBI, SLR, GPS and GLONASS), the parameters are therefore seasonal fluctuations are comparable, as in the off-site co-located instruments should measure the same physical motion of the Earth. Any discrepancy between the determined parameters of seasonal fluctuations in this case can be interpreted as techno-specific errors on the same time interval. In addition, the network of observing stations, as a result of global processing, equalized and stabiliziruemost the accuracy of the observations. Given the comparability of seasonal variation in data obtained by different methods, we used for the analysis of VLBI observations with the telescope RT-22.

VLBI is a method created for observations in astrophysics and astrometry. Later this method was implemented in geodesy depending on the ground and celestial target observation sessions in terms of the number of observed radio sources, as well as the number and sequence of observations for radio sources. Radio telescopes that form the source lines in a network of stations are fairly stably attached to the underlying rock through their foundations. The surface of the Earth, however, is unstable. In addition, the lithosphere is subject to various deformations. Some of the deformations are fairly constant or periodic. Others are individual, episodic and intermittent, for example, during and after a seismic event.

We studied periodic processes in the data on the insolation of the earth's surface at Kara-Dag using the time-frequency continuous wavelet analysis, the results of which are shown in Fig.1.

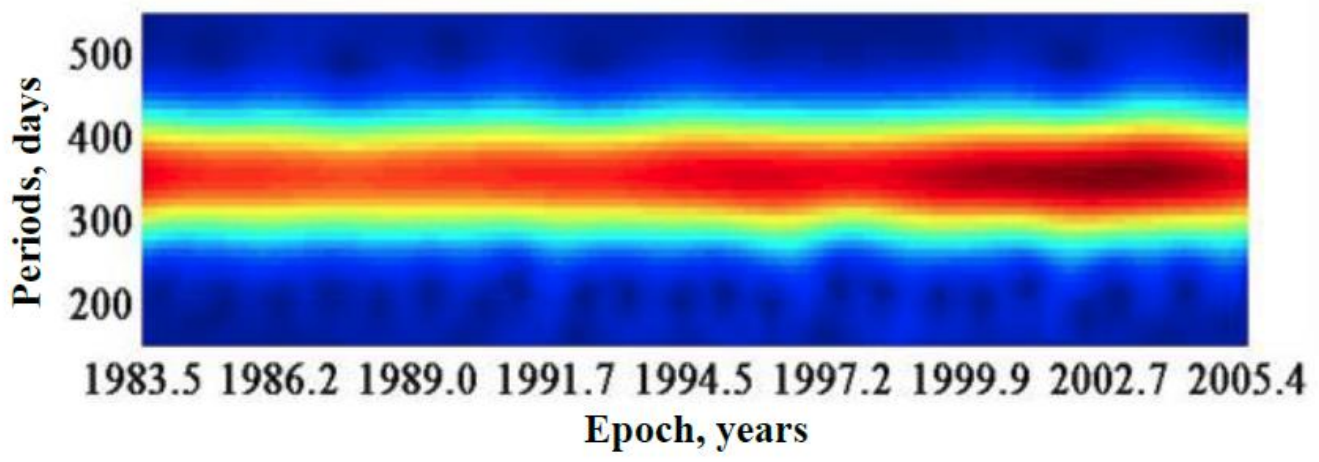

Fig. 1. Continuous time-frequency wavelet analysis ('morl' wavelets) of data.

Together with geodynamics, oceanography, glaciology, meteorology and climatology, geodesy provides a metric basis for interdisciplinary research within the framework of Earth science.

The transportation of masses in the global geophysical liquid and gaseous shells of the Earth (atmosphere, oceans, hydrology, tides, mantle, core) affects changes in the Earth's rotation parameters, causes changes in the gravitational field and displacement of the geocenter. In addition, under the influence of lunar-solar tides, the Earth's surface is irreversibly deformed. In addition to predictable deformations, there are changes that are not explainable in the theory of tides, the so-called unmodeled additives to deformations of the surface of solid earth. Currently, the problem of achieving millimeter accuracy in determining the positions and displacement rates of observational instruments requires taking these additives into account, which has become possible due to the coordinated work in the network of observation stations [3]. 


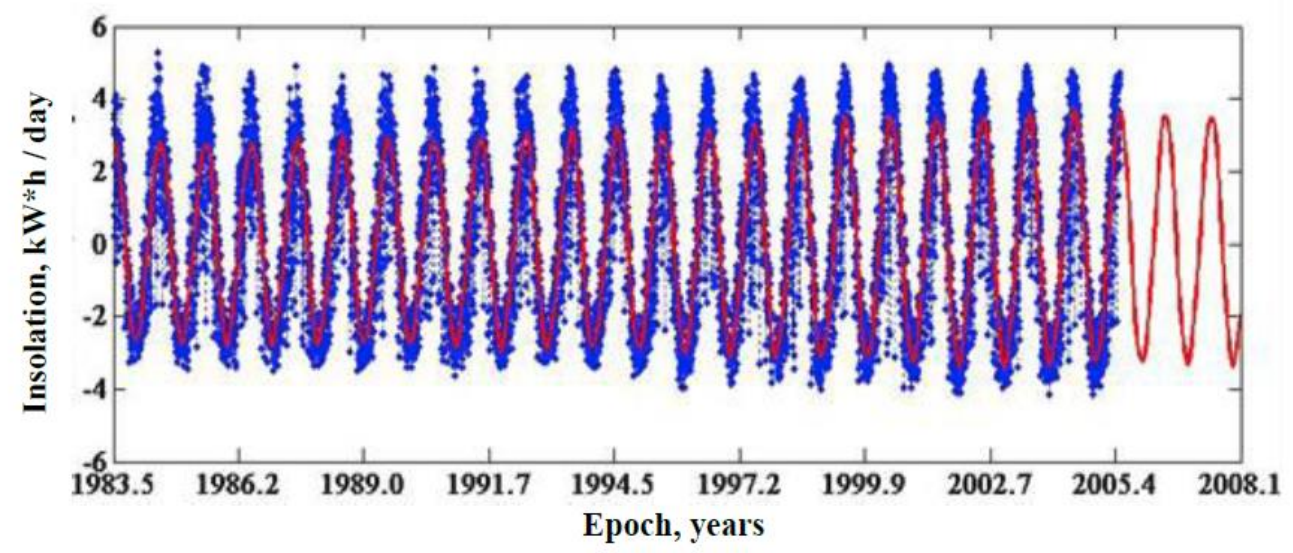

Fig. 2. Data on the insolation of the earth's surface at Kara-Dag for the period 1983-2005 after the removal of irregular "white noise" (marked with asterisks *); approximation curve of the periodic component in the data (solid line) calculated using a 6-order sinusoidal model. After 2005.4 - forecast.

The amplitude of the most significant oscillations with periods $365.3,365.7$ days, respectively equal to 6.34 and $4.8 \mathrm{~kW} * \mathrm{~h} /$ day. The amplitudes of the remaining periodic oscillations in model of 6 order are within error of approximate data. For example, the amplitude of the semiannual oscillation with a period of 182.2 days equal to $0.2 \mathrm{~kW} * \mathrm{~h} /$ day. It follows that the underlying periodic process (the seasonal component) is the sum of two out of phase waves with periods of $\sim 365$ days $[4,5]$.

\section{Conclusion}

1. The local study of regular and irregular changes of the earth's surface of the Crimea, and also to accompanying the failure strain of various objects of livelihoods and environmental degradation, is necessary in connection with the progressive increase in various kinds of critical deformations of the ground surface of the Crimea. Held for many years at the site "SimeizKatsively" of observations according to agreed international programs currently provide the basis for conducting both academic and applied research. At the same time, the position of Crimea near the rift of the Eurasian plate and the increased development of the Peninsula building complexes requires the creation of an integrated network of local geodynamic stations, prediction capabilities, and environmental safety for carrying out geodetic, construction and agricultural works. The study of local irregular changes in shape of the Earth, gravity, magnetic and seismic fields, local and general deformations of the earth's crust is necessary for earthquake forecasting by identifying the area with abnormal values of these parameters.

2. In addition to the predictable deformation, there are changes not explainable in the theory of the tides, so-called non-simulated supplements to the deformation of the solid surface of the earth. Currently, the problem of achieving millimeter precision in determining the positions and velocities of displacements of the Supervisory tools requires consideration of these additives, made possible by a coordinated network of observing stations. Due to the daily rotation of the Earth and motion of Earth, moon and Sun in their orbits tide-generating force at each point on the Earth's surface continuously changing in time, never exactly repeating. The earth is not absolutely rigid body under the action of external forces, the distance between two arbitrary points is changed. If the action of external forces ceases, then the points will not be returned to its original position, i.e., the Land remains in a deformed state. We obtained information on seasonal additions to the deformation of the earth at the VLBI station Simeiz is used to refine the seasonal displacements of the coordinates of the station. 
3. The growth of the oscillation amplitude in the seasonal component from the data of insolation falling on the surface of the earth in the Crimea can be explained by the process of addition of two oscillations with periods of $\sim 365$ days. At the same time, regular excess insolation of the earth's surface in the Kara-Dag in comparison with other towns of Crimea may be associated exclusively with structural properties of the Kara Dag mountain. The difference is that after removing periodic fluctuations in the data about insolation of the earth's surface at the point of the Kara-Dag there are traces of short-term energy effects in the low frequency region after 1986 and after 1995, possibly related to the extreme reaction of the geological structure of the mountain Kara-Dag on the space exposure.

\section{References}

1. Sidorenkov N. S., Atmospheric processes and rotation of the Earth. Gidrometeoizdat, Spb (2002)

2. Sidorenkov N. S., Priroda. 2, 23-31 (2008)

3. Kurbasova G.S., Volvach A. E., and Volvach L. N., Cosmic Research, 57, 4,243 (2019)

4. Volvach A. E., Kurbasova G. S., Volvach L. N. Astrophysical Bulletin, 74, 3, 331 (2019)

5. Volvach A.E., Kurbasova G.S., Earth physics, (to be published) 